of these compounds, the results confirmed that the addition of multiple sulfo groups to PSTs reduces the compounds' binding affinities to voltage-gated sodium channels. This strongly suggests that sulfo groups reduce PST toxicity, further highlighting their potential for incorporation into PST-based drug candidates.

The use of biosynthetic enzymes to modify PSTs represents a strategy that is distinct from the chemical-synthesis approaches more frequently used to make analogues of these natural products ${ }^{12}$. Although many of those synthetic efforts have been successful, they often involve long sequences of reactions and deliver low yields of products as a consequence of the challenging architectures of the PSTs - which contain an abundance of reactive oxygen and nitrogen atoms that complicate the use of more-standard chemical reactions. Lukowski and colleagues' findings now offer researchers the opportunity to combine conventional synthetic chemistry with biocatalysis, using enzymes to further modify PST scaffolds obtained by synthetic routes. This could potentially streamline access to sulfated versions of these natural products. It might eventually even be possible

to use this approach to make non-natural PST analogues for evaluation as candidate therapeutics.

However, substantial barriers must be surmounted before these sulfotransferase enzymes can be fully integrated into PST syntheses. Their catalytic efficiency is very low, and they have not yet been used on a large scale - Lukowski

"These findings offer researchers the opportunity to combine conventional synthetic chemistry with biocatalysis." and colleagues worked at a submilligram scale, but multi-gram quantities of PST analogues would eventually be needed for the preclinical development of drug candidates. Also, the reactivity of the enzymes towards non-natural PST scaffolds, or towards members of related toxin families, has yet to be evaluated. If the reactivity and selectivity of the sulfotransferases can be optimized using enzyme engineering, these biocatalysts will become powerful synthetic tools in the search for new pain therapeutics.
Monica E. McCallum and Emily P. Balskus are in the Department of Chemistry and Chemical Biology, Harvard University, Cambridge, Massachusetts 02138, USA. e-mail:balskus@chemistry.harvard.edu

1. Durán-Riveroll, L. M. \& Cembella, A. D. Mar. Drugs 15, 303 (2017).

2. Lukowski, A. L. et al. ACS Chem. Biol. 14, 941-948 (2019).

3. van Apeldoorn, M. E., van Egmond, H. P., Speijers, G. J. A. \& Bakker, G. J. I. Mol. Nutr. Food Res. 51, 7-60 (2007).

4. Bustillos-Guzmán, J. J. et al. Food Addit. Contam. A 32, 381-394 (2015).

5. Andrinolo, D., Michea, L. F. \& Lagos, N. Toxicon 37, 447-464 (1999).

6. Andrinolo, D., Iglesias, V., Garcia, C. \& Lagos, N. Toxicon 40, 699-709 (2002).

7. Sako, Y. et al. J. Phycol. 37, 1044-1051 (2001)

8. Yoshida, T. et al. Fish. Sci. 68, 634-642 (2002).

9. Wang, D.-Z., Zhang, S.-F., Zhang, Y. \& Lin, L. J. Proteom. 135, 132-140 (2016).

10.Cullen, A. et al. ACS Chem. Biol. 13, 3107-3114 (2018).

11.Lukowski, A. L. et al. J. Am. Chem. Soc. 140, 11863-11869 (2018).

12.Berlinck, R. G. S., Bertonha, A. F., Takaki, M. \& Rodriguez, J. P. G. Nat. Prod. Rep. 34, 1264-1301 (2017).

This article was published online on 10 June 2019.

\title{
A licence to kill during inflammation
}

Inflammasomes are protein complexes that fight infection by driving inflammation or cell death. It now seems that the protein NEK7 provides a 'licence' for the formation of inflammasomes containing the protein NLRP3. SEE ARTICLE P.338

\section{KENGO NOZAKI \& EDWARD A. MIAO}

$\mathrm{I}$ nflammation can help to eliminate infection, but excessive inflammation can cause damage to the body. The sensor proteins that trigger an inflammatory immune response must therefore be carefully regulated. Some intracellular immune-sensor proteins detect components in a cell that become abnormal or altered during a cellular crisis. Signs of cellular crisis are sometimes produced in the absence of an infection, so mechanisms are needed to prevent the proteins from triggering an inappropriate inflammatory response. Sharif et al. ${ }^{1}$ report a structural study on page 338 that investigates an immune-sensor protein called NLRP3, revealing that a protein called NEK7 acts as a 'licence' that enables this protein to cause inflammation.

When an immune sensor recognizes a hallmark of infection in the cytoplasm, this can activate the protein and lead to the assembly of a multiprotein complex called an inflammasome. The activation of proteins that function downstream of an inflammasome can potently drive both inflammation and cell death ${ }^{2}$. Different types of inflammasome can form depending on the sensor components involved. Certain inflammasomes respond to a highly specific trigger: for example, those in mammalian cells containing the sensor protein NLRC4 respond to the presence of the bacterial protein flagellin ${ }^{3,4}$.

Proteins that are normally present in mammalian cells do not seem able to trigger the accidental formation of NLRC4-containing inflammasomes, given the lack of reports of such aberrant events. By contrast, inflammasomes that contain NLRP3 are activated when NLRP3 recognizes - by an as yet unknown mechanism - hallmarks of cellular catastrophe, such as extremely low concentrations of potassium in the cytoplasm, or signs of dysfunction in organelles called mitochondria ${ }^{2}$. Such events can arise from tissue damage that is unrelated to infection, and NLRP3 activation in such cases has been implicated as a possible cause of inflammatory diseases such as atherosclerosis.
It is widely accepted that the tightly regulated formation of NLRP3-containing inflammasomes occurs in two steps. In the first step, NLRP3 is primed for action by other immune-sensor proteins called TLRs, which can detect components of microorganisms. This priming step occurs in two ways ${ }^{2}$ : NLRP3 can undergo a modification, such as the addition of a phosphate group or the removal of an attached ubiquitin protein. Further priming is achieved by a rise in expression of the gene that encodes NLRP3, increasing the chance that NLRP3 will detect any abnormalities. The second step, activation, then results in NLRP3 proteins binding together to form part of a disc-shaped inflammasome complex that is probably similar to those of other inflammasomes containing proteins of the NLR family (which includes NLRP3 and NLRC4) (5, $^{5}$. This activation step occurs during a cellular catastrophe, but the biochemical and structural mechanisms involved are unknown.

Researchers have long sought to determine the structure of NLRP3 as it forms an inflammasome, in the hope of gaining insights into how this protein functions. However, such efforts have been unsuccessful, perhaps because unknown protein partners that interact with NLRP3 were missing from earlier attempts.

The discovery ${ }^{7-9}$ that the enzyme NEK7 is essential for NLRP3 signalling provided a missing part of the puzzle. NEK7 regulates processes that occur during cell division, such as the breakdown of the nuclear-envelope structure ${ }^{10}$, so it was surprising to find that it has a separate role in inflammation. This suggested that NLRP3-containing inflammasome formation doesn't occur during cell division because NEK7 


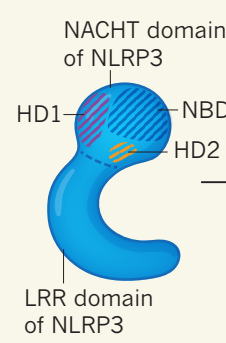

Licence revoked

during cell division

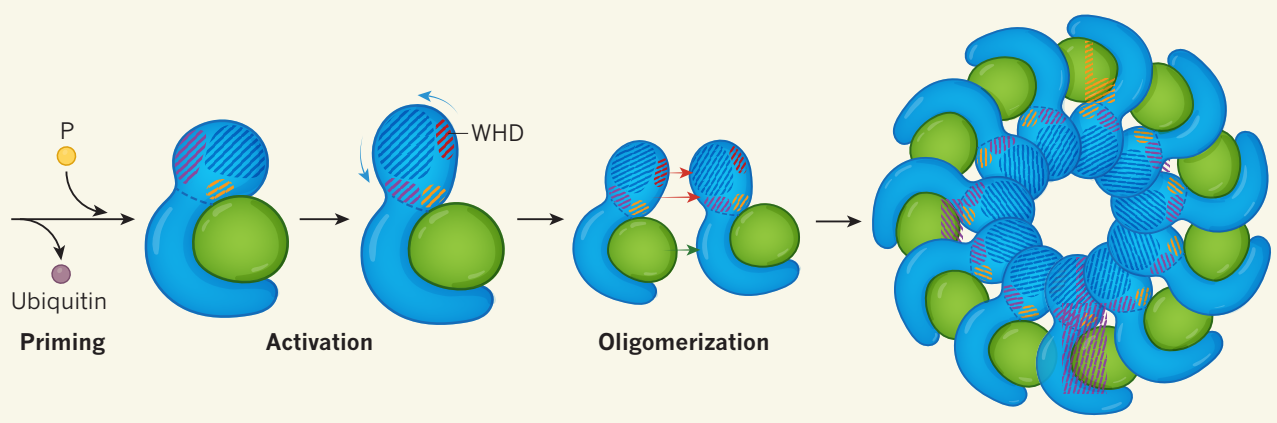

Inflammasome formation causes inflammation or cell death
Figure 1 | The NEK7 protein enables the formation of a multiprotein immune-defence complex called the inflammasome. Sharif et al. ${ }^{1}$ report structural studies of the assembly of an inflammasome containing the human protein NLRP3. Inflammasome formation requires ${ }^{7-9}$ the enzyme NEK7. Sharif and colleagues report that NEK7 helps the inflammasome to assemble by providing a 'licence' for its formation. Given that NEK7 has roles in cell division, this licensing activity is probably revoked if NEK7 is not available when cells divide. NLRP3 has a NACHT domain composed of the subdomains: NBD, HD1, WHD and HD2 (the WHD subdomain is exposed when the protein is activated) and an LRR domain. In the inactive state of NLRP3, NEK7 binds to
NLRP3's LRR domain and to its NACHT domain in the HD2 subdomain. An early step leading to inflammasome formation is called priming, which is when NLRP3 either gains a phosphate group $(\mathrm{P})$ or loses an attached ubiquitin protein ${ }^{2}$. This is followed by rotational activation of the NACHT domain, which exposes all four of its subdomains. By analogy with another type of inflammasome ${ }^{5,6}$, three of these subdomains (NBD, HD1 and WHD) might form interactions (red arrows) between adjacent proteins. Sharif et al. report that NEK7 forms a connection (green arrow) between LRR domains of adjacent NLRP3 proteins as the oligomerization of proteins occurs during inflammasome formation. The assembled inflammasome can cause inflammation or cell death. is unavailable to aid inflammasome assembly ${ }^{8}$. NEK7 regulates NLRP3 signalling by binding to a region of the protein known as the LRR domain $^{8,9}$. However, why such an interaction between NEK7 and NLRP3 is essential for inflammasome formation has remained elusive.

To tackle this question, Sharif et al. used cryo-electron microscopy to investigate the structure of a human NLRP3 interacting with NEK7. The authors' structural data reveal that NLRP3 and NEK7 bind to form a dimer in which NLRP3 is in an inactive conformation. In this state, the LRR domain of NLRP3 has a lobed, semicircular shape (Fig. 1), and the carboxy-terminal region of NEK7 nestles in the inner curve of the LRR.

NLRP3 also contains a structure called the NACHT domain, and in the inactive NLRP3-NEK7 complex, this domain is structurally very similar to the NACHT domain ${ }^{11}$ of inactive NLRC4. It was previously shown $n^{5,6,11}$ that the NACHT domain of NLRC4 rotates as it transitions into an active conformation. This rotational-activation step uncovers part of the NACHT surface, enabling inflammasome formation through a protein-assembly process called oligomerization, and generating a discshaped NLRC4-containing inflammasome $e^{5,6}$. The authors used this information to model a hypothetical conformation for an NLRP3containing inflammasome.

In Sharif and colleagues' model, the hypothetical rotational activation of NLRP3 doesn't affect NEK7 binding, and NEK7 still fits into NLRP3's LRR domain in the same way as in the inactive structure. Furthermore, the authors made the surprising discovery that NEK7 provides a bridge between adjacent NLRP3 proteins, by forming an interface with the LRR of the adjacent NLRP3 in the inflammasome. The ability to form such an interface suggests that NEK7 provides a licence for NLRP3 to form part of the inflammasome. It seems that this licensing event occurs independently of both the priming and rotational-activation steps, because the authors did not include molecules that cause priming or add triggers for rotational activation in their structural studies. The results suggest a revised view of how the NLRP3containing inflammasome is regulated, and put forward the idea that NLRP3 oligomerization requires NEK7 licensing. Taken together “The assembled
inflammasome
can cause
inflammation,
or cell death." with evidence from earlier studies ${ }^{7-9}$, it seems likely that this licence is revoked during cell division.

The function of NEK7 in the NLRP3containing inflammasome is interesting when considered in relation to the structure of the NLRC4-containing inflammasome. The LRR domain of NLRC4 is longer than that of NLRP3, and makes direct contact with the LRR of the adjacent NLRC4 protein in the inflammasome ${ }^{5,6}$. In an NLRP3-containing inflammasome, NEK7 fulfils a similar connecting role by making contact with adjacent LRR domains. This explains why NLRP3-containing inflammasomes require NEK7 licensing, whereas NLRC4-containing ones do not.

Many mysteries concerning the regulation of NLRP3-containing inflammasomes remain. Perhaps future structural studies will reveal how NLRP3 modification accomplishes the priming step.

Finally, we still don't know the answer to perhaps the most important question of all: what direct interaction between NLRP3 and an unknown cellular factor results in the formation of the inflammasome? Perhaps the evidence that the NEK7 licence is revoked during cell division provides a clue. If inappropriate activation of NLRP3 is likely to occur during cell division, then having an NEK7-licensing step would help to combat this potential problem. Thus, one could imagine that the type of cellular catastrophe detected by NLRP3 also occurs during cell division but in a controlled manner.

Kengo Nozaki and Edward A. Miao are in the Department of Microbiology and Immunology, Center for Gastrointestinal Biology and Disease, and at the Lineberger Comprehensive Cancer Center, University of

North Carolina at Chapel Hill, Chapel Hill,

North Carolina 27599, USA.

e-mail:edmiao1@gmail.com

1. Sharif, H. et al. Nature 570, 338-343 (2019).

2. Swanson, K. V., Deng, M. \& Ting, J. P.-Y. Nature Rev Immunol. https://doi.org/10.1038/s41577-0190165-0 (2019).

3. Miao, E. A. et al. Nature Immunol. 7, 569-575 (2006).

4. Franchi, L. et al. Nature Immunol. 7, 576-582 (2006).

5. Zhang, L. et al. Science 350, 404-409 (2015)

6. Hu, Z. et al. Science 350, 399-404 (2015).

7. Schmid-Burgk, J. L. et al. J. Biol. Chem. 291 103-109 (2016).

8. Shi, H. et al. Nature Immunol. 17, 250-258 (2016).

9. He, Y., Zeng, M. Y., Yang, D., Motro, B. \& Núñez, G. Nature 530, 354-357 (2016).

10.Fry, A. M., Bayliss, R. \& Roig, J. Front. Cell Dev. Biol. 5, 102 (2017).

11. Hu, Z. et al. Science 341, 172-175 (2013).

This article was published online on 12 June 2019. 